ORIGINAL ARTICLE

\title{
INVESTIGATION OF THE PARAMETERS THAT MAY PREDICT HEMODIALYSIS FREQUENCY
}

\author{
Gökhan Kıral ${ }^{1}$, Alperen Taha Certel ${ }^{1}$, Irmak İrem Özyiğit ${ }^{1}$, Begüm Söyleyici $^{1}$, Güray Aygün ${ }^{2}$, Cenk Gökalp ${ }^{3}$ \\ ${ }^{1}$ Trakya University School of Medicine, Edirne, TURKEY \\ ${ }^{2}$ Department of Internal Medicine, Trakya University School of Medicine, Edirne, TURKEY \\ ${ }^{3}$ Division of Nephrology, Department of Internal Medicine Trakya University School of Medicine, Edirne, TURKEY
}

\begin{abstract}
Aims: This study investigates the relationship between the clinical parameters at the beginning of hemodialysis therapy and the changing in the frequency of weekly hemodialysis sessions in ongoing treatment. Methods: The study population was composed of all of the patients that were started chronic hemodialysis treatment between January 2015 and January 2020. The patients were classified as twice-weekly (2/7) and thrice-weekly (3/7) groups according to the hemodialysis schedule at the start of treatment. The $3 / 7$ group is additionally subdivided according to the dialysis schedule switches to the thrice-weekly program. Basal demographics and biochemical parameters were obtained from medical records at the start of hemodialysis. Results: The total number of subjects in the study was 433 (141 in the twice-weekly program, 292 in the thrice-weekly program). Forty-six (32.6\%) patients in twice-weekly hemodialysis program were shifted to a thrice-weekly program during the follow-up. The female/ male ratio in the $2 / 7$ program is different from the $3 / 7$ program. Serum creatinine and C-reactive protein levels were higher in patients on the $3 / 7$ program. Serum calcium and albumin levels were higher in patients on a 2/7 program. The statistically significant increments were found in calcium, hemoglobin, hematocrit, urea, creatinine, and potassium levels between the before and after switch results of the 3/7 group which switched from 2/7. Conclusion: Urea, creatinine, and potassium levels can guide the decision to compose a hemodialysis schedule. However, patients' future weekly treatment schedules cannot be predicted by biochemical parameters obtained at the start of hemodialysis. Keywords: Hemodialysis, dialysis frequency, chronic kidney disease, biochemical parameters
\end{abstract}

\section{INTRODUCTION}

Chronic kidney disease (CKD) is present in 9.1\% of the world population, and approximately $10 \%$ of those patients have a glomerular filtration rate (GFR) less than $15 \mathrm{~mL} / \mathrm{min} / \mathrm{m}^{2}$ (1). Hemodialysis (HD) is a well known and widely distributed renal replacement modality (RRT) modality worldwide. Despite the advances in hemodialysis techniques, patients on hemodialysis have higher mortality rates and lower quality of life than transplantation and peritoneal dialysis $(2,3)$. Hemodialysis modality has some disadvantages such as requiring the specified health care professionals, patients' dependency on a dialysis center three times a week with at least four hours in a day, and the increase in long-term cost that is a big burden to the health care system. Hemodialysis may be planned as a twice-weekly schedule considering to minimize all these disad- vantages with patients who had the acceptable urinary output and biochemical evaluation.

Herein, we investigated the biochemical and clinical parameters that may affect the long term dialysis schedule in the patients who started the chronic dialysis program.

\section{MATERIAL AND METHODS}

\section{PATIENT SELECTION}

The study protocol was approved by the Trakya University Ethical Board (Protocol Code: TÜTFBAEK 2020-345). Four hundred thirty-three patients that were started hemodialysis between January 2015 to January 2020 have been enrolled in this retrospective study. Inclusion criteria were being more than 
18 years old and hemodialysis history for more than three months. Exclusion criteria were being younger than 18 years of age. Trakya University Hospital, as a tertiary medical facility, is one of the major hospitals in the Thrace region of Turkey and it serves high level nephrology care around 800,000 population. The patients were classified according to the dialysis schedule twice-weekly and thrice-weekly at the time of hemodialysis initiation. The twice-weekly group was classified into two subgroups that were ongoing with a twice-weekly schedule and switched to thrice-weekly schedule subgroups.

Age, gender, hemodialysis program, comorbidities, CKD ethiologies, urea, creatinine, uric acid, electrolyte levels, complete blood count, and C-reactive protein (CRP) values at the start of dialysis were recorded for statistical analysis.

\section{STATISTICAL ANALYSIS}

Categorical data are presented as frequencies and percentages. Continuous variables are expressed as mean \pm standard deviation (SD) for normally distributed variables and as the median and interquartile range (IQR, 25th-75th percentile) for variables with skewed distribution. The Kolmogorov-Smirnov test was used to test for a normal distribution. The differences between groups were analyzed using the Student $t$-test and the Mann-Whitney $U$ test as appropriate. A p-value of $<0.05$ was considered significant. Statistical analysis was performed using SPSS version 23.0 (SPSS Inc., Chicago, IL, USA).

\section{RESULTS}

A total of 433 patients were included in this retrospective study. One hundred and forty-one (32.6\%) patients were started twice-weekly HD schedule, and 292 (67.4\%) patients were started thrice-weekly HD schedule at baseline. Forty-six (32.6\%) patients in twice-weekly HD program were shifted to a thrice-weekly program during the follow-up. The mean age of all patients was 64.4 years, and $179(41.3 \%)$ were female. The female to male ratio was higher in twice-weekly program group besides lower in the thrice-weekly program group (Table 1). Basal serum calcium and albumin levels were significantly higher in the twice-weekly group. Basal serum creatinine and CRP levels were significantly higher in the thrice-weekly group. Other basal demographic and biochemical parameters did not differ be- tween the two treatment groups.

There was no demographic and biochemical difference observed between the patients who continued with the twice-weekly program and shifted the thrice-weekly program during the follow-up (Table 2).

The parameters at the start of the twice-weekly $\mathrm{HD}$ program and at the time of the switching the HD schedule to thrice-weekly were compared to elucidate the impact of biochemical parameters on the increase of HD frequency (Table 3 ). There were statistically significant differences in urea, creatinine, potassium, calcium, hemoglobin, and hematocrit values observed.

\section{DISCUSSION}

Chronic kidney disease is a worldwide health problem that significantly impacts patient morbidity and mortality (1). At the most severe CKD stage, called end-stage renal disease (ESRD), the patients need either dialysis or transplantation to survive. Hemodialysis is the most common renal replacement therapy applied in the whole world (4). Hemodialysis is generally received with a thrice-weekly schedule because of the limited supply of the dialysis centers, increasing demand for dialysis, and economic issues. Attachment to a dialysis center on certain days of the week, typical side effects like fatigue, nausea, vomiting, muscle cramps on dialysis days, a stricter diet, and increased mortality compared to other modalities are some disadvantages for HD treatment (5). In some HD patients, a twice-weekly HD program is sufficient for effective solute removal and volume control. In general, the ESRD patients who had preserved urine output, adequate solute clearance and water removal may have a chance to receive a twice-weekly schedule.

In this study, we investigated if any parameters, besides urine output, correlate the HD need at the beginning of the HD treatment in the future. There were more female patients in the twice-weekly group, but male patients predominate the whole study population. Data obtained from the Chronic Renal Insufficiency Cohort (CRIC)-Study showed a significantly lower risk for ESRD development in the female community (6). Our study showed that the females develop less end-stage renal disease and have better residual renal functions. The lower serum albumin and calcium levels were detected in the thrice-weekly group while better nutritional status and less-severe bone-mineral disorders in the twice-weekly group. Serum CRP was higher in the thrice-weekly group. This supports the previous 
Table 1: Demographics and biochemical parameters at the start of hemodialysis treatment.

\begin{tabular}{|c|c|c|c|}
\hline & $\begin{array}{l}\text { Twice-weekly } \\
\text { hemodialysis } \\
\quad(n=141)\end{array}$ & $\begin{array}{l}\text { Thrice-weekly } \\
\text { hemodialysis } \\
(n=292)\end{array}$ & $P$-value* \\
\hline Gender [n (\%)] & & & 0.002 \\
\hline Female & $73(52 \%)$ & $106(36 \%)$ & \\
\hline Male & $68(48 \%)$ & $186(64 \%)$ & \\
\hline Age (years) & $66.04 \pm 12.91$ & $65.08 \pm 13.62$ & 0.516 \\
\hline Hypertension [n (\%)] & $95(67 \%)$ & $191(65 \%)$ & 0.330 \\
\hline Etiology of CKD & & & 0.234 \\
\hline Diabetes Mellitus & $7(5 \%)$ & $13(4 \%)$ & \\
\hline Hypertension & $3(2 \%)$ & $11(4 \%)$ & \\
\hline Glomerulonephritis & $13(9 \%)$ & $13(4 \%)$ & \\
\hline TIN & $4(3 \%)$ & $7(2 \%)$ & \\
\hline PKD & $5(4 \%)$ & $8(2 \%)$ & \\
\hline Amyloidosis & $1(1 \%)$ & $7(2 \%)$ & \\
\hline Others/Unknown & $108(76 \%)$ & $233(82 \%)$ & \\
\hline Urea (mg/dL) & $113.13 \pm 45.16$ & $108.12 \pm 46.33$ & 0.207 \\
\hline Creatinine (mg/dL) & $4.95 \pm 1.69$ & $5.78 \pm 2.28$ & 0.001 \\
\hline Uric Acid (mg/dL) & $5.53 \pm 1.77$ & $5.73 \pm 1.83$ & 0.303 \\
\hline $\mathrm{Na}^{+}(\mathbf{m E q} / \mathbf{L})$ & $136.83 \pm 3.76$ & $136.29 \pm 3.96$ & 0.356 \\
\hline $\mathbf{K}^{+}(\mathbf{m E q} / \mathbf{L})$ & $4.40 \pm 0.58$ & $4.51 \pm 0.66$ & 0.101 \\
\hline $\mathrm{Ca}^{+2}(\mathrm{mEq} / \mathrm{L})$ & $8.48 \pm 0.83$ & $8.29 \pm 0.86$ & 0.004 \\
\hline $\mathrm{PO}_{4}^{-2}(\mathrm{mEq} / \mathrm{L})$ & $4.64 \pm 1.17$ & $4.86 \pm 1.40$ & 0.111 \\
\hline Albumin (g/dL) & $3.09 \pm 0.60$ & $2.93 \pm 0.68$ & 0.017 \\
\hline CRP (mg/dL) & $3.11 \pm 0.39$ & $4.48 \pm 0.37$ & 0.004 \\
\hline Hemoglobin (g/dL) & $9.42 \pm 1.26$ & $9.35 \pm 1.21$ & 0.530 \\
\hline Hematocrit (\%) & $27.85 \pm 4.05$ & $27.80 \pm 3.99$ & 0.783 \\
\hline
\end{tabular}

CKD: Chronic Kidney Disease, TIN: Tubulointerstitial Nephritis, PKD: Polycystic Kidney Disease, CRP: C-reactive protein

*Statistically significant values are marked as bold. 
Table 2: Demographics and parameters of twice-weekly program patients.

Ongoing twice-weekly Switched to thrice-weekly $P$-value $(n=95) \quad(n=46)$

\begin{tabular}{lccc}
\hline Gender [n (\%)] & & $27(58 \%)$ & 0.208 \\
Female & $44(46 \%)$ & $19(42 \%)$ & \\
Male & $51(54 \%)$ & $65.54 \pm 11.69$ & 0.588 \\
Age (years) & $66.14 \pm 13.48$ & $105.69 \pm 40.874$ & 0.264 \\
Urea (mg/dL) & $116.47 \pm 40.02$ & $4.99 \pm 1.58$ & 0.733 \\
Creatinine (mg/dL) & $4.93 \pm 1.74$ & $5.63 \pm 1.82$ & 0.781 \\
Uric Acid (mg/dL) & $5.48 \pm 1.76$ & $136.91 \pm 3.93$ & 0.893 \\
Na ${ }^{+}$(mEq/L) & $136.80 \pm 3.71$ & $4.34 \pm 0.59$ & 0.410 \\
$\mathbf{K}^{+}$(mEq/L) & $4.40 \pm 0.56$ & $8.41 \pm 0.80$ & 0.165 \\
Ca $^{+2}$ (mEq/L) & $8.51 \pm 0.85$ & $4.54 \pm 0.95$ & 0.627 \\
PO4 ${ }^{-2}$ (mEq/L) & $4.69 \pm 1.27$ & $3.08 \pm 0.60$ & 0.954 \\
Albumin (g/dL) & $3.09 \pm 0.60$ & $3.28 \pm 0.75$ & 0.968 \\
CRP (mg/dL) $_{\text {Hemoglobin (g/dL) }}$ & $3.06 \pm 0.48$ & $9.20 \pm 1.39$ & 0.180 \\
Hematocrit (\%) & $9.50 \pm 1.17$ & $27.68 \pm 4.57$ & 0.690 \\
\hline
\end{tabular}

CRP: C-reactive protein

Table 3: Biochemical parameters at the beginning and at the switching of twice-weekly patients.

Beginning with a twice-weekly plan
Switched to the thrice- $\quad P$-value* weekly plan

\begin{tabular}{lccc}
\hline Urea $(\mathbf{m g} / \mathbf{d L})$ & $102.16 \pm 41.37$ & $121.92 \pm 43.87$ & $\mathbf{0 . 0 0 9}$ \\
Creatinine (mg/dL) & $4.99 \pm 1.58$ & $6.22 \pm 2.17$ & $<\mathbf{0 . 0 0 1}$ \\
Uric Acid (mg/dL) & $5.59 \pm 1.77$ & $5.9 \pm 1.54$ & 0.517 \\
$\mathbf{N a}^{+}(\mathbf{m E q} / \mathbf{L})$ & $136.82 \pm 4.10$ & $137.72 \pm 3.67$ & 0.212 \\
$\mathbf{K}^{+}(\mathbf{m E q} / \mathbf{L})$ & $4.37 \pm 0.63$ & $4.76 \pm 0.77$ & $\mathbf{0 . 0 0 8}$ \\
$\mathbf{C a}^{+\mathbf{2}}(\mathbf{m E q} / \mathbf{L})$ & $8.41 \pm 0.82$ & $8.84 \pm 0.76$ & $\mathbf{0 . 0 0 4}$ \\
PO4 $^{-\mathbf{2}}(\mathbf{m E q} / \mathbf{L})$ & $4.49 \pm 0.95$ & $4.57 \pm 1.48$ & 0.744 \\
Hemoglobin $(\mathbf{g} / \mathbf{d L})$ & $9.20 \pm 1.41$ & $10.06 \pm 1.76$ & $\mathbf{0 . 0 1 9}$ \\
Hematocrit (\%) & $27.66 \pm 4.57$ & $30.37 \pm 5.27$ & $\mathbf{0 . 0 1 6}$
\end{tabular}

*Statistically significant values are marked as bold. 
studies about increased microinflammation negatively correlated with residual renal functions in the HD population (7).

We could not observe any significant difference between thrice-weekly and twice-weekly groups comparing basal parameters. These findings support that the evaluation of residual renal function and urine output had a major role in the decision-making process of scheduled HD sessions.

While we compare the basal and shifting biochemical parameters, we observed a statistically significant increase in urea, creatinine, and potassium levels at the time of rescheduling. This can be explained by a decrease in residual kidney function in some twice-weekly patients with time. So not just in the start of HD treatment but also in the follow-up period, close monitoring of some biochemical parameters may make the doctors aware of the patients who need more frequent HD sessions.

In conclusion, hemodialysis schedule can be decided by urea, creatinine, and potassium levels. However, patients' future weekly treatment schedules cannot be foreseen by biochemical parameters obtained at the start of hemodialysis.

Ethics Committee Approval: This study was approved by the Scientific Research Ethical Committee of Trakya University School of Medicine (Protocol Code: TÜTF-BAEK 2020-345).

Informed Consent: N/A

Conflict of Interest: The authors declared no conflict of interest. Author contributions: Concept: CG Design: CG Supervision: CG Resources: GK, ATC, IIIÖ, BG, GA, CG Materials: CG Data collection and/or processing: GK, ATC, IIÖ, BG Analysis and/or Interpretation: GA, CG, GK, ATC, IİÖ, BG Literature Search: GA, CG, GK, ATC, IIÖ, BG Writing Manuscript: GA, CG Critical Review: GA, CG, GK, ATC, IİÖ, BG.

Financial disclosure: The authors declared that this study received no financial support.

Editor in chief's Note: Three of the authors of this article, Alperen Taha Certel, Irmak İrem Özyiğit, and Begüm Söyleyici are members of the editorial board of Turkish Medical Student Journal. However, they did not take place in any stage of the editorial decision of the manuscript. The editors who evaluated this manuscript are from other institutions.

\section{REFERENCES}

1. Carney EF. The impact of chronic kidney disease on global health. Nat Rev Nephrol 2020;16(5):251.

2. Rambod M, Shabani M, Shokrpour N et al. Quality of life of hemodialysis and renal transplantation patients. Health Care Manag 2011;30(1):23-8.

3. Jung HY, Jeon Y, Park Y et al. Better quality of life of peritoneal dialysis compared to hemodialysis over a two-year period after dialysis initiation. Sci Rep 2019;9(1):10266.

4. Ng JK, Li PK. Chronic kidney disease epidemic: How do we deal with it? Nephrology 2018;23(4):116-20.

5. Li H, Xie L, Yang J et al. Symptom burden amongst patients suffering from end-stage renal disease and receiving dialysis: A literature review. Int J Nurs Sci 2018;5(4):427-31.

6. Ricardo AC, Yang W, Sha D et al. Sex-related disparities in CKD progression. J Am Soc Nephrol 2019;30(1):137-46.

7. de Sequera P, Corchete E, Bohorquez L et al. Residual renal function in hemodialysis and inflammation. Ther Apher Dial 2017;21(6):592-8. 